\title{
Assessing the impact of a school program of early detection of asthma
}

\author{
Luciano Busi ${ }^{1}$, Sabrina Fernández ${ }^{1}$, and Peter Sly $^{2}$ \\ ${ }^{1}$ Trelew Hospital \\ ${ }^{2}$ University of Queensland
}

June 23, 2020

\begin{abstract}
Background: Wheeze-associated disorders are common in childhood, associated with considerable morbidity, if not detected and treated. Under diagnosis of asthma remains a problem, especially in resource-limited settings. Methods: We used a validated school-based screening questionnaire to detect children likely to have asthma. Children with positive screening were referred to the Pulmonology Department for clinical review and lung function testing. We compared asthma-like symptoms, activity limitation, school absence and health service utilization before and after in those diagnosed with, and treated for asthma. Results: 6400 children, from a potential population of 70,000 were screened between 2010 and 2016, with 900 (14.1\%) screening positive. Lung function data were available from $578(64.2 \%)$ children (5.7 to 6.5 years old). Asthma was confirmed in 549 children; 438 were treated with short acting bronchodilator alone and 111 with inhaled corticosteroids. Asthma control improved in $58 \%$ of children, with fewer daytime [mean 4.7 (SD1.9), vs 11.1 (0.6) days per week, p<0.001] and nocturnal [4.3 (1.1) vs 0.89 (0.5) days per month, $\mathrm{p}<0.001]$ symptoms. Activity improved and fewer school days were lost due to asthma [12.8 (3.0) vs 1.9 (0.9) days in past 3 months, $\mathrm{p}<0.001]$ in over $50 \%$ of children. Emergency department visits were reduced [1.8 (0.7) vs $0.3(0.2)$ visits in past 3 months, $\mathrm{p}<0.001]$ in over $80 \%$ of children. Conclusions: Asthma under diagnosis remains a problem in Argentina. Our school-based assessment is an effective tool for detecting children with undiagnosed asthma. Instituting effective asthma treatment in these children reduces symptoms and improves control.
\end{abstract}

Assessing the impact of a school program of early detection of asthma

Luciano E Busi MD ${ }^{1,2}$, Sabrina G Fernández $\mathrm{MD}^{2,3}$, Peter D Sly MD, $\mathrm{DSc}^{4}$

${ }^{1}$ Pulmonology Committee of the Argentinean Pediatric Society, Argentina.

${ }^{2}$ Trelew Hospital, Argentina.

${ }^{3}$ Puerto Madryn Hospital, Argentina.

${ }^{4}$ Children's Health and Environment Program, Child Health Research Centre, University of Queensland, Brisbane, Australia.

Funding: Trelew Hospital, Argentina.

Address for Correspondence: lucianobusi2@gmail.com

Keywords: disease screening, questionnaire, Argentina, children

Running title: School-based asthma screening

Summary/Abstract (249/250 words) 
Background: Wheeze-associated disorders are common in childhood, associated with considerable morbidity, if not detected and treated. Under diagnosis of asthma remains a problem, especially in resource-limited settings.

Methods: We used a validated school-based screening questionnaire to detect children likely to have asthma. Children with positive screening were referred to the Pulmonology Department for clinical review and lung function testing. We compared asthma-like symptoms, activity limitation, school absence and health service utilization before and after in those diagnosed with, and treated for asthma.

Results: 6400 children, from a potential population of 70,000 were screened between 2010 and 2016, with 900 $(14.1 \%)$ screening positive. Lung function data were available from $578(64.2 \%)$ children (5.7 to 6.5 years old). Asthma was confirmed in 549 children; 438 were treated with short acting bronchodilator alone and 111 with inhaled corticosteroids. Asthma control improved in $58 \%$ of children, with fewer daytime [mean 4.7 (SD1.9), vs 11.1 (0.6) days per week, $\mathrm{p}<0.001$ ] and nocturnal [4.3 (1.1) vs 0.89 (0.5) days per month, $\mathrm{p}<0.001]$ symptoms. Activity improved and fewer school days were lost due to asthma [12.8 (3.0) vs 1.9 (0.9) days in past 3 months, $\mathrm{p}<0.001$ ] in over $50 \%$ of children. Emergency department visits were reduced [1.8 (0.7) vs $0.3(0.2)$ visits in past 3 months, $\mathrm{p}<0.001$ ] in over $80 \%$ of children.

Conclusions: Asthma under diagnosis remains a problem in Argentina. Our school-based assessment is an effective tool for detecting children with undiagnosed asthma. Instituting effective asthma treatment in these children reduces symptoms and improves control.

\section{Introduction}

Wheezing disorders in children, frequently called asthma (1), are common causes of morbidity and mortality in children. The world has seen an epidemic increase in asthma in recent decades, starting in high income countries and proceeding to middle and low income countries (2). Seminal studies conducted as part of the International Study of Asthma and Allergies in Children (ISAAC) have documented changes in prevalence in various part of the world between the late 1990s and mid 2000s (3-5). The prevalence reported for Latin American countries showed considerable variability (3-5), with that reported for Argentina being around 10 to $20 \%$.

Despite improved diagnosis and management in many parts of the world, under diagnosis of asthma is still a major problem, especially in impoverished communities and in low and middle income countries (LMICs) $(2,6-8)$. This is especially true for young children, despite specific guidelines produced for the under five year olds (9). Under diagnosis is frequently associated with inappropriate management and increased morbidity including school absence and disturbance of family work practices with consequent economic consequences for the family and society $(6,7)$. The need for timely and accurate diagnosis in young children is recognized (7), is considered as a research priority (10), and forms part of a recently published Children's Asthma Charter that sets out the rights and expectations of children with asthma (11). Appropriate diagnosis and management leads to improved outcomes for children and their families (7).

Wheeze and asthma-like symptoms are common in preschool-aged children and around $80 \%$ of those with persistent asthma have symptoms originating in early life (12). However, only approximately $30 \%$ of preschoolers with recurrent wheeze will go on to have childhood asthma (12). The consequence of not having an accurate diagnosis is often inappropriate treatment with antibiotics or with inhaled corticosteroids plus long-acting beta agonists $(9,13,14)$. Appropriate diagnosis at early school age with appropriate management according to guidelines is likely to improve outcome in individual children and reduce the need for health care utilization $(7,14,15)$.

Very few studies worldwide (and none in Latin America) have evaluated the impact of the programs designed to screen for asthma in children in the early school years. We developed and validated programs for early detection of under-diagnosed or under-treated asthmatic children attending the first grade of school (16) or 
kindergarten (17). The aim of the present study was to determine the impact of early diagnosis and treatment of asthma in school-aged children in Chubut, Argentina.

\section{Material and methods}

The program, known locally as PDPACh (Programa de Detección Precoz de Asma en escuelas de la Provincia de Chubut [Early School Case-Detection Asthma Program in Chubut province]), aids early detection of asthma in first grade school children in Chubut province, Argentina (16). Parents completed a previously validated questionnaire (Table 1) and children with a positive result were referred to the Pulmonology Department, Trelew Hospital, for assessment. The study protocol was approved by the Bioethics Committee and Committee for Teaching and Research, Trelew Hospital and Parents gave written consent for their child's participation.

Data were collected from children referred for asthma assessment before and after parents completed PDPACh. We compared data from the 3 months prior to receiving the questionnaire with the 3 months after in terms of: 1) number of days per week with daytime symptoms; 2) number of days per month with nocturnal symptoms; 3) number of days per week with restriction of usual activity; 4) number of lost school days due to asthma in the 3 months period; 5) number of visits to an emergency service due to asthma symptoms; 6 ) asthma control using the Asthma Control Test and classified according to the Global Initiative for Asthma (GINA) criteria; and 7) lung function.

\section{Lung function}

Spirometry was performed following ATS guidelines modified for children (18) by the same technician on all occasions. Forced expiratory volumes and flows were reported, together with the response to bronchodilator for appropriate outcome variables. Lung function outcome variables were reported as Z-scores using the GLI Caucasian equations (19), as validated for Argentinian children (20).

Statistical analysis

Comparisons before and after assessment were made using paired-t tests, chi-square and ANOVA using SPSS version 23.0. Statistical significance was accepted at the $5 \%$ level.

Table 1. Parent Questionnaire (PQ) (translated). The original Spanish version is available in Busi et al. (16)

Does your child ..

Never Some

Develop coughs that won't go away?

Wake up at night because of difficulty breathing?

Have a hard time taking a Deep breath?

Make noisy or wheezy sounds when breathing (awake)?

Complain about a chest that feels tight or hurts after running, playing hard or doing sports?

Wake up at night coughing?

Cough after running, climbing stairs or playing sports?

Miss days of school (absent from school) because of breathing problems?

Has a doctor or nurse told you that your child has asthma, reactive airways disease or wheezy bronchitis?

No

No

Has your child stayed in the hospital overnight for asthma or for trouble breathing?

Does your child take medicine or use an inhaler for asthma?

\section{Results}

During the period between 2010 and 2016 the questionnaire was completed by parents of approximately 6400 children from a total potential population of approximately 70,000 children (9.1\%). For the present study, 
we attempted to contact 900 families of children with a positive screening questionnaire (14.1\%). Study data were retrieved for $578(64.2 \%)$ children (5.7 to 6.5 years old) referred for asthma assessment from whom lung function data were available. Asthma was confirmed in 549 children, 438 were treated with short acting bronchodilator alone and 111 with inhaled corticosteroids.

On grouped data, marked improvements were seen in all of the clinical variables assessed, in asthma control and in lung function (Table 2). Asthma control was improved in $58 \%$ of children, with fewer daytime and nocturnal symptoms and improved activity and fewer days off school due to asthma in more than $50 \%$ of children. Emergency department visits were reduced in more than $80 \%$ of children.

Table 2: Asthma-like symptoms, activity limitation, health service utilization and asthma control in the 3 months before and after asthma diagnosis and treatment.

Variable

Days per week with daytime symptoms. Mean (SD)

Days per month with nocturnal symptoms. Mean (SD)

Days per week of activity limitation. Mean (SD)

Days off school due to asthma past 3 months. Mean (SD)

ED visits for asthma past 3 months. Mean (SD)

Asthma control: Well controlled

Partly controlled asthma

Uncontrolled asthma

Lung function* FVC (z-score), mean (SD) $\mathrm{FEV}_{1}$ (z-score), mean (SD) FEV 0.75 (z-score), mean (SD) FEF $25-75$ (z-score), me

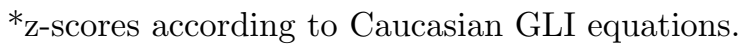

\section{Discussion}

The results from the present study confirm previous reports on the benefits of achieving an appropriate asthma diagnosis in young children. Those in whom asthma was diagnosed and treated appropriately following a positive screening questionnaire (16) showed improvements in lung function, reduction in symptoms, reduced health care service utilization and improved asthma control. On an individual basis, 318 (58\%) children had improved asthma control, 289 (50\%) children missed fewer day of school and 439 (80\%) children had fewer visits to the emergency department for asthma-like symptoms.

Under diagnosis of asthma remains a major problem, especially in resource limited situations. The recently published "Worldwide Charter for Children with Asthma" (11) recognizes this issue as a major problem for children globally. The first item listed under the heading "Rights of Children with Asthma" is 'I deserve a timely and accurate diagnosis of asthma within the primary care/community setting'. (11). As outlined by a recent Concise Clinical Review (21) "Under diagnosis can occur either because the patient has not communicated his/her symptoms to a physician, because the physician has not assigned a diagnosis to explain the patient's symptoms, or because the physician has attributed the patient's respiratory symptoms to a condition other than asthma." In the LMIC setting one might add "where the family does not have access to appropriate health care" and in the pediatric setting, the failure to recognize asthma-like symptoms in young children further compounds the problem (9). The results from the present study show that under diagnosis can be a major problem in children in the early school years. Fourteen percent (900/6400) of children returned a positive screening questionnaire. We were able to contact and obtain data from 578 children and $95 \%(549 / 578)$ of these were diagnosed with and treated for asthma. Even if those families whose children had a very positive outcome from the screening questionnaire were more likely to respond to our contact attempts, this is still a remarkable outcome. Even if the 322 children whose families did not respond did not benefit, this still leaves 61\% (549/900) children with major benefits from appropriate asthma diagnosis and treatment following the positive screening questionnaire. Extrapolating further, assuming the children of the 6,400 families completing the questionnaire were representative of the potential total 70,000 
population, a positive screen rate of $14 \%(6,400 / 70,000)$ would see 9,800 children return a positive screening questionnaire, with between 5978 ( at a rate of 61\%) and 9310 (at a rate of $95 \%$ ) under diagnosed and under treated asthmatic children.

In summary, under diagnose and under treatment of asthma in the early school years remains a major problem in Argentina. The program of early detection of asthma with questionnaires in the province of Chubut, Argentina is an effective instrument to identify under diagnosed or undertreated asthmatic children and reduced morbidity, improve lung function and school attendance and reduce health care service utilization in young children.

\section{References}

1. Pavord ID, Beasley R, Agusti A, Anderson GP, Bel E, Brusselle G, et al. After asthma: redefining airways diseases. Lancet. 2018;391:350-400.

2. Dharmage SC, Perret JL, Custovic A. Epidemiology of Asthma in Children and Adults. Frontiers in Pediatrics. 2019;7:246.

3. Worldwide variation in prevalence of symptoms of asthma, allergic rhinoconjunctivitis, and atopic eczema: ISAAC. The International Study of Asthma and Allergies in Childhood (ISAAC) Steering Committee. Lancet. 1998;351:1225-32.

4. Asher MI, Montefort S, Bjorksten B, Lai CK, Strachan DP, Weiland SK, et al. Worldwide time trends in the prevalence of symptoms of asthma, allergic rhinoconjunctivitis, and eczema in childhood: ISAAC Phases One and Three repeat multicountry cross-sectional surveys. Lancet. 2006;368:733-43.

5. Sole D, Mallol J, Camelo-Nunes IC, Wandalsen GF. Prevalence of rhinitis-related symptoms in Latin American children - results of the International Study of Asthma and Allergies in Childhood (ISAAC) phase three. Pediatric Allergy and Immunology. 2010;21(1 Pt 2):e127-36.

6. Cruz AA, Stelmach R, Ponte EV. Asthma prevalence and severity in low-resource communities. Current opinion in allergy and clinical immunology. 2017;17(3):188-93.

7. Fischer GB, Sarria EE, Camargos P, Mocelin HT, Soto-Quiroz M, Cruz AA, et al. Childhood asthma in low and middle-income countries: Where are we now? Paediatric Respiratory Reviews. 2019;31:52-7.

8. Kjaergaard J, Anastasaki M, Stubbe Ostergaard M, Isaeva E, Akylbekov A, Nguyen NQ, et al. Diagnosis and treatment of acute respiratory illness in children under five in primary care in low-, middle-, and highincome countries: A descriptive FRESH AIR study. PloS one. 2019;14(11):e0221389.

9. Pedersen SE, Hurd SS, Lemanske RF, Becker A, Zar HJ, Sly PD, et al. Global strategy for the diagnosis and management of asthma in children 5 years and younger. Pediatric Pulmonology. 2010;46:1-17.

10. Mathioudakis AG, Custovic A, Deschildre A, Ducharme FM, Kalayci O, Murray C, et al. Research Priorities in Pediatric Asthma: Results of a Global Survey of Multiple Stakeholder Groups by the Pediatric Asthma in Real Life (PeARL) Think Tank. The Journal of Allergy and Clinical Immunology In Practice. 2020 .

11. Szefler SJ, Fitzgerald DA, Adachi Y, Doull IJ, Fischer GB, Fletcher M, et al. A worldwide charter for all children with asthma. Pediatric Pulmonology. 2020;55(5):1282-92.

12. Castro-Rodriguez JA. The necessity of having asthma predictive scores in children. Journal of Allergy and Clinical Immunology. 2013;132(6):1311-3.

13. AIHW:, Correll P, Poulos L, Ampon R, Reddel H, Marks G. Respiratory Medicaton use in Australia. Canberra: AIHW; 2015. Contract No.: Cat. no. ACM31.

14. Australian Centre for Asthma Monitoring. Asthma in Australian children: findings from Growing up in Australia, the longitudinal study of Australian children. Canberra: AIHW; 2009. 
15. Reddel HK, Bateman ED, Becker A, Boulet LP, Cruz AA, Drazen JM, et al. A summary of the new GINA strategy: a roadmap to asthma control. European Respiratory Journal. 2015;46(3):622-39.

16. Busi LE, Sly PD, Restuccia S, Llancamán L. Validation of a school-based written questionnaire for asthma case identification in Argentina. Pediatric Pulmonology. 2012;47(1):1-7.

17. Busi LE, Sly PD, Llancaman L. Validation of a questionnaire for asthma case identification in pre-schools in Latin America. Respirology. 2015;20(6):912-6.

18. Arets HGM, Brackel HJL, van der Ent CK. Forced expiratory manoeuvres in children: do they meet ATS and ERS criteria for spirometry? European Respiratory Journal. 2001;18(4):655.

19. Quanjer PH, Stanojevic S, Cole TJ, Baur X, Hall GL, Culver BH, et al. Multi-ethnic reference values for spirometry for the 3-95-yr age range: the global lung function 2012 equations. European Respiratory Journal. 2012;40(6):1324-43.

20. Busi LE, Sly PD. Validation of the GLI-2012 spirometry reference equations in Argentinian children. Pediatric pulmonology. 2018;53(2):204-8.

21. Aaron SD, Boulet LP, Reddel HK, Gershon AS. Underdiagnosis and Overdiagnosis of Asthma. American Journal of Respiratory and Critical Care Medicine. 2018;198(8):1012-20. 